\title{
Adding the s-Process Element Cerium to the APOGEE Survey: Identification and Characterization of Ce II Lines in the H-band Spectral Window
}

\author{
Katia Cunha ${ }^{1,2}$, Verne V. Smith ${ }^{3}$, Sten Hasselquist ${ }^{4}$, Diogo Souto ${ }^{2}$, Matthew D. Shetrone ${ }^{5}$, Carlos Allende Prieto ${ }^{6,7}$, \\ Dmitry Bizyaev $^{8,9}$, Peter Frinchaboy ${ }^{10}$, D. Anibal García-Hernández ${ }^{6,7}$, Jon Holtzman ${ }^{11}$, Jennifer A. Johnson ${ }^{12}$, Henrik Jónsson ${ }^{6,7}$, \\ Steven R. Majewski ${ }^{13}$, Szabolcs Mészáros ${ }^{14,19}$, David Nidever ${ }^{3}$, Mark Pinsonneault ${ }^{12}$, Ricardo P. Schiavon ${ }^{15}$, Jennifer Sobeck ${ }^{13}$, \\ Michael F. Skrutskie ${ }^{16}$, Olga Zamora ${ }^{6,7}$, Gail Zasowski ${ }^{17}$, and J. G. Fernández-Trincado ${ }^{18}$ \\ ${ }^{1}$ University of Arizona, Tucson, AZ 85719, USA \\ ${ }^{2}$ Observatório Nacional, São Cristóvão, Rio de Janeiro, Brazil; kcunha@on.br \\ ${ }^{3}$ National Optical Astronomy Observatories, Tucson, AZ 85719, USA \\ ${ }^{4}$ Department of Astronomy, New Mexico State University, Las Cruces, NM 88003, USA \\ ${ }^{5}$ Department of Astronomy and McDonald Observatory, University of Texas, Austin, TX 78712, USA \\ ${ }^{6}$ Instituto de Astrofísica de Canarias, La Laguna, 38200 Tenerife, Spain \\ ${ }^{7}$ Departamento de Astrofísica, Universidad de La Laguna, 38200 Tenerife, Spain \\ ${ }^{8}$ Apache Point Observatory, Sunspot, NM 88349, USA \\ ${ }^{9}$ Sternberg Astronomical Institute, Moscow State University, Moscow 119992, Russia \\ ${ }^{10}$ Texas Christian University, Dallas, TX, USA \\ ${ }^{11}$ Department of Astronomy, New Mexico State University, Las Cruces, NM 88003, USA \\ ${ }_{12}$ Department of Astronomy, Ohio State University, Columbus, OH 43210, USA \\ ${ }^{13}$ Department of Astronomy, University of Virginia, Charlottesville, VA 22904, USA \\ ${ }^{14}$ ELTE Gothard Astrophysical Observatory, H-9700 Szombathely, Szent Imre Herceg st. 112, Hungary \\ Astrophysics Research Institute, Liverpool John Moores University, Liverpool, L3 5RF, UK \\ ${ }^{16}$ Department of Astronomy, P.O. Box 400325, 530 McCormick Road, Charlottesville, VA 22904-4325, USA \\ ${ }^{17}$ STScI, Baltimore, MD, 21218, USA and Dept of Physics \& Astronomy, University of Utah, Salt Lake City, UT, 84112, USA \\ ${ }^{18}$ Departamento de Astronomía, Universidad de Concepción, Casilla 160-C, Concepción, Chile \\ Received 2017 February 16; revised 2017 June 13; accepted 2017 June 14; published 2017 August 1
}

\begin{abstract}
Nine Ce II lines have been identified and characterized within the spectral window observed by the Apache Point Observatory Galactic Evolution Experiment (APOGEE) survey (between $\lambda 1.51$ and $1.69 \mu \mathrm{m}$ ). At solar metallicities, cerium is an element that is produced predominantly as a result of the slow capture of neutrons (the s-process) during asymptotic giant branch stellar evolution. The Ce II lines were identified using a combination of a high-resolution $(R=\lambda / \delta \lambda=100,000)$ Fourier Transform Spectrometer (FTS) spectrum of $\alpha$ Boo and an APOGEE spectrum $(R=22,400)$ of a metal-poor, but s-process enriched, red giant (2M16011638-1201525). Laboratory oscillator strengths are not available for these lines. Astrophysical $g f$-values were derived using $\alpha$ Boo as a standard star, with the absolute cerium abundance in $\alpha$ Boo set by using optical Ce II lines that have precise published laboratory $g f$-values. The near-infrared Ce II lines identified here are also analyzed, as consistency checks, in a small number of bright red giants using archival FTS spectra, as well as a small sample of APOGEE red giants, including two members of the open cluster NGC 6819, two field stars, and seven metal-poor N- and Al-rich stars. The conclusion is that this set of Ce II lines can be detected and analyzed in a large fraction of the APOGEE red giant sample and will be useful for probing chemical evolution of the s-process products in various populations of the Milky Way.
\end{abstract}

Key words: stars: abundances

\section{Introduction}

The Apache Point Observatory Galactic Evolution Experiment (APOGEE) is one of the programs within the third and fourth stages of the Sloan Digital Sky Survey (SDSS III, Eisenstein et al. 2011; SDSS-IV, Blanton et al. 2017). APOGEE is a near-infrared (NIR), high-resolution $(R \sim$ $22,500)$ spectroscopic survey in an $\mathrm{H}$-band spectral window that by 2020 will have observed $\sim 400,000$ stars, primarily red giants from all major Galactic stellar populations (targeting strategies are described in Zasowski et al. 2013). From 2011-2014, as part of SDSS III, APOGEE observed over 130,000 individual stars (Majewski et al. 2017). Spectra covering the wavelength range from $\lambda 15100 \AA$ to $\lambda 16900 \AA$ are obtained with a cryogenic, 300 fiber spectrograph using the $2.5 \mathrm{~m}$ SDSS telescope (Gunn et al. 2006; Wilson et al. 2010).

\footnotetext{
${ }^{19}$ Premium Postdoctoral Fellow of the Hungarian Academy of Sciences, Hungary.
}

In 2017, the APOGEE survey will deploy a second spectrograph on the du Pont $2.5 \mathrm{~m}$ telescope to give access to southern hemisphere targets. From raw APOGEE spectra, an automated data reduction pipeline (Nidever et al. 2015) produces one-dimensional, wavelength-calibrated, flux-calibrated spectra, with terrestrial airglow and atmospheric absorption lines removed.

Stellar parameters and chemical abundances are obtained from the APOGEE spectra using the APOGEE Stellar Parameters and Chemical Abundances Pipeline (ASPCAP; García Pérez et al. 2016). ASPCAP uses an extensive grid of synthetic spectra to match to the observed spectra, with final stellar parameters and chemical abundances derived from the best fits to the observed spectra using the FERRE $^{20}$ code (Allende Prieto et al. 2006) for interpolation within a grid of library spectra (Zamora et al. 2015). Construction of the library

\footnotetext{
${ }^{20}$ FERRE is available from https://github.com/callendeprieto/ferre.
} 
rests upon an extensive and detailed list of atomic and molecular spectral lines needed to model the spectra of red giant stars to high accuracy and standard plane-parallel and spherical model atmospheres in local thermodynamic equilibrium (LTE; Mészáros et al. 2012 and references therein).

Although the APOGEE line list represents a comprehensive set of both atomic and molecular lines in the H-band, it is affected by the fact that $g f$-values of many transitions in the infrared either have not been measured in the laboratory or have large uncertainties (Wahlgren et al. 2008). In the absence of accurately measured or predicted values, APOGEE has also used astrophysical $g f$-values, which are derived from spectroscopic analyses of two standard stars, the Sun and $\alpha$ Boo, but keeping the $g f$-values of the molecular lines of $\mathrm{CO}, \mathrm{CN}$, and $\mathrm{OH}$ with their original laboratory-based $g f$-values. The details of the construction of the APOGEE/ASPCAP line list are described in Shetrone et al. (2015). Part of this process involved a hybrid approach, constraining atomic $g f$-values based upon both laboratory and theoretical values, in combination with syntheses of the solar spectrum and the spectrum of the well-studied K-giant $\alpha$ Boo. Such a hybrid approach has achieved a good level of both internal and external accuracy in abundance scales $(<\sim 0.1 \mathrm{dex}$, in most cases), as demonstrated by comparisons with abundance results based upon optical studies of open clusters from the literature (see the discussion in Holtzman et al. 2015).

Improving the APOGEE/ASPCAP line list is an ongoing endeavor, with upgrades and modifications taking place regularly. Initial tests of an early version of the APOGEE line list were carried out by Smith et al. (2013). This manual abundance analysis of a small sample of selected field $\mathrm{K}$ and $\mathrm{M}$ giants confirmed that there were numerous missing lines from the APOGEE line list. (See Shetrone et al. 2015 for a list of then unidentified lines in the APOGEE region.) The line list used to generate the most recent data release, DR13, yielded a set of elemental chemical abundances for red giant stars that includes C, N, O, Na, Mg, Al, Si, S, K, Ca, Ti, V, Cr, Mn, Fe, $\mathrm{Co}$, and $\mathrm{Ni}$. Although this line list includes elements from many of the important nucleosynthesis processes that drive chemical evolution in the Galaxy, noticeably absent are heavy, neutron-rich elements $(Z \geqslant 30)$, which include mostly those nuclei produced by neutron captures via both the s- and r-process. Although abundances for one heavy element ( $\mathrm{Rb}$; $Z=37$ ) are reported in the latest data release (DR13), these results are based upon very weak lines of $\mathrm{Rb}$ I that have not been scrutinized closely to date; such abundances should be viewed with caution. One s-process element with a high density of absorption lines expected in the spectrum of red giant stars is cerium, which presents a fairly low ionization energy $(5.54 \mathrm{eV})$ and thus is expected to be mostly ionized in the atmospheres of red giant stars. Cerium is an excellent element with which to probe the second peak main s-process; in the solar system, Ce consists primarily of two stable isotopes $\left(88.5 \%\right.$ as ${ }^{140} \mathrm{Ce}$ and $11.1 \%$ as ${ }^{142} \mathrm{Ce}$ ), and the s-process accounts for $\sim 90 \%$ of it, with the r-process contributing the other $\sim 10 \%$ and very minor contributions $(\sim 0.4 \%)$ from the p-process nuclei ${ }^{136} \mathrm{Ce}$ and ${ }^{138}$ Ce (Sneden et al. 2008; Lodders 2010).

This paper reports the first identification of a sample of Ce II lines within the APOGEE spectral window, along with the characterization of their $g f$-values via an optical abundance analyses of $\alpha$ Boo, and constraints from the solar spectrum. The $g f$-values derived are then used to obtain $\mathrm{Ce}$ abundances in the four red giants analyzed by Smith et al. (2013) and in a small sample of field and cluster red giants observed by APOGEE. Cerium abundances are also derived and discussed for an interesting sample of N-rich and Al-rich stars, one of which was found to be s-process enhanced. All of these stars and their respective $\mathrm{Ce}$ abundance determinations aid in constraining and vetting the astrophysical $g f$-values derived here for the $\mathrm{Ce}$ II lines.

\section{The Ce II Lines in the APOGEE Region}

\subsection{Identification of s-process Lines in the APOGEE Spectra}

To reliably identify missing lines from the APOGEE line list, it is important to investigate their observed behavior as a function of stellar parameters and metallicity. In particular, stars known to be enriched in s-process elements are useful tools for isolating potential s-process lines, in comparison to stars with more common chemical compositions.

A variety of stellar spectra were employed to identify potential s-process lines in the APOGEE window: the $\alpha$ Boo infrared spectral atlas by Hinkle et al. (1995), along with archival stellar spectra observed with the KPNO 4 m telescope and the Fourier Transform Spectrometer (FTS) of two nearsolar-metallicity M-giants, $\beta$ And and $\delta$ Oph, as well as the metal-rich K-giant $\mu$ Leo, and the asymptotic giant branch (AGB) star HD 199799 (all previously analyzed in Smith et al. 2013 and collectively referred to in this paper as "FTS standards"). These high-resolution spectra ( $R=100,000$ for $\alpha$ Boo and $R \sim 40,000$ for the other stars) are suitable for identifying possible s-process lines, while the stars themselves also span a range of $T_{\text {eff }}$ and metallicity that is representative of major stellar components observed by the APOGEE survey. Within this sample of stars, HD 199799 is of particular importance as it is a thermally pulsing AGB star, with enhanced s-process elements (including the short-lived radioactive s-process element technetium; Smith \& Lambert 1990). In addition, the metal-poor $([\mathrm{Fe} / \mathrm{H}]=-1.4)$, very $\mathrm{N}$ - and $\mathrm{Al}$ rich, red giant 2M16011638-1201525, which was observed by APOGEE and studied by Fernández-Trincado et al. (2016), exhibits many of the unidentified, potential s-process lines very strongly. The spectrum of this star was particularly valuable because the lines in this metal-poor giant are weak, enhancing the contrast with s-process lines, making this star crucially important in securing identifications of s-process lines.

A number of the unidentified candidate s-process lines in the APOGEE spectra were found to be Ce II lines based on wavelength comparisons with the extensive laboratory spectroscopic study of the second spectrum of cerium (Ce II) by Corliss (1973). In addition to Ce II, a number of other unidentified lines were found to be Nd II lines, based on the spectroscopic study of Blaise et al. (1970), and these are discussed in a companion paper by Hasselquist et al. (2016). Table 1 provides a list of nine Ce II lines identified in the APOGEE region (in air wavelengths), including one Ce II line $(\lambda 15829.83 \AA)$ that falls in a spectral gap between the blue and green APOGEE chips and is presented in Table 1 for completeness. The relative emission-line intensities of the nine Ce II lines, along with their respective excitation potentials $(\chi)$ and energy levels from Corliss (1973), agreed with their relative strengths in the observed spectra of red giants. The $g f$-values for the Ce II lines, however, are not provided in the 
Table 1

Identified Ce II Lines

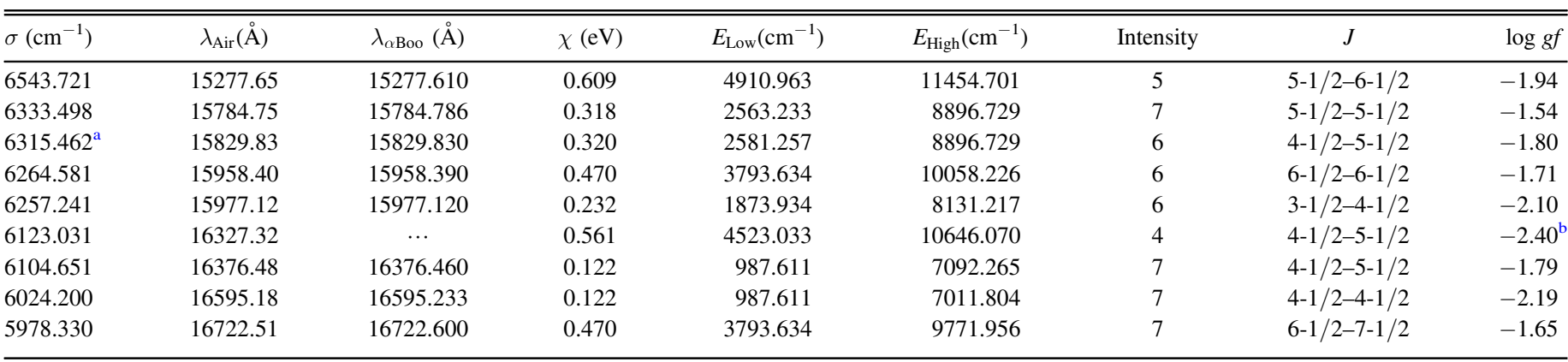

Notes.

${ }^{a}$ This line falls between APOGEE detectors.

b This $g$-value was measured using 2M16011638-1201525.

Corliss (1973) study; they have also not been found in literature searches nor in the NIST database. ${ }^{21}$

Most of the Ce II lines in the APOGEE window are well defined in the $\mathrm{K} 2$ giant $\alpha \mathrm{Boo}$, even though this red giant is mildly metal poor $([\mathrm{Fe} / \mathrm{H}]=-0.52)$ and not s-process enhanced, while these same lines are rather weak, or not measurable at all, in the solar spectrum. The solar spectrum can be used, however, to constrain the $g f$-values of the underlying lines found in the spectral regions of interest, as well as to provide some general confirmation for the derived $g f$-values for the Ce II lines for a solar Ce abundance of $1.58 \pm 0.04$ (Grevesse et al. 2015). Given the line excitation potentials from Corliss (1973) and a Ce abundance in $\alpha$ Boo based on optical Ce II lines (Section 2.2), astrophysical $g f$-values can be derived for the sample of APOGEE Ce II lines identified here. The metal-poor s-process rich red giant 2M16011638-1201525 was also used to set the $g f$-value for one Ce II line that is dominated by blends in $\alpha$ Boo and also to test the self-consistency of the astrophysical $g f$-values derived from $\alpha$ Boo.

\subsection{The Cerium Abundance in $\alpha$ Boo from Optical Lines}

The cerium abundance in $\alpha$ Boo was derived using the visible spectral atlas from Hinkle et al. (2000) and the 2012 version of the 1D LTE code MOOG (Sneden 1973). Rather than rely on literature values, a more self-consistent procedure is to use optical Ce II lines with measured $g f$-values and small uncertainties to determine the $\mathrm{Ce}$ abundance in $\alpha$ Boo using the same ATLAS9 model atmospheres (Kurucz 1993) as used in the construction of the APOGEE line list, which is from the grid computed for ASPCAP by Mészáros et al. (2012). All subsequent calculations in this study also use MOOG with the Kurucz ATLAS9 models. The adopted stellar parameters for $\alpha$ Boo were taken from Ramirez \& Allende Prieto (2011; Table 4), and we note that these were also the stellar parameters used in the construction of the ASPCAP line list.

Table 2 summarizes information on the four Ce II lines used in the optical analysis of $\alpha$ Boo. The $g f$-values for these transitions were taken from Lawler et al. (2009). The derived abundances for each Ce II line were set by spectrum synthesis, with nearby atomic lines (from the Kurucz ${ }^{22}$ line list) and CN lines (from Davis \& Phillips 1963) included in the synthesis (although none of the four Ce II lines are

\footnotetext{
21 https://physics.nist.gov/PhysRefData/ASD/lines_form.html

22 http://www.cfa.harvard.edu/amp/ampdata/kurucz23/sekur.html
}

Table 2

Optical Ce II Lines in $\alpha$ Boo

\begin{tabular}{lcccc}
\hline \hline$\lambda_{\text {Air }}(\AA)$ & $\chi(\mathrm{eV})$ & $\log g f$ & $\alpha$ Boo EqW $(\mathrm{m \AA})$ & $A(\mathrm{Ce})$ \\
\hline 5274.23 & 1.04 & +0.13 & 34.0 & 0.98 \\
5330.56 & 0.87 & -0.40 & 18.7 & 0.94 \\
5975.82 & 1.33 & -0.45 & 5.8 & 0.97 \\
6043.37 & 1.21 & -0.48 & 9.2 & 1.05 \\
\hline
\end{tabular}

measurably affected by blends). Equivalent widths were also measured, and these are presented in Table 2 only as an indication of the strengths of the sampled Ce II lines. The mean $\mathrm{Ce}$ abundance and standard deviation obtained is $A$ $(\mathrm{Ce})=0.99 \pm 0.05$; the line-to-line scatter is very close to the uncertainties in the $g f$-values themselves, indicating that these lines and high-quality oscillator strengths yield a selfconsistent cerium abundance in $\alpha$ Boo.

The same Ce II lines used to set the cerium abundance in $\alpha$ Boo were also analyzed in an optical spectrum of 2M160116381201525 taken with the Apache Point Observatory $3.5 \mathrm{~m}$ echelle spectrograph at a resolution $R=30,000$. The stellar parameters for this star were derived previously (see Hasselquist et al. 2016). The Ce abundance obtained from the optical lines is $A$ $(\mathrm{Ce})=1.22 \pm 0.06$, and this is compared to the Ce abundance derived from the Ce II lines in the APOGEE window based upon the astrophysical $g f$-values in Section 2.3.

\subsection{Astrophysical gf-values}

The astrophysical $g f$-values of Ce II lines in the APOGEE window were obtained using both the $\alpha$ Boo IR spectral atlas by Hinkle et al. (1995; $R=100,000)$, along with the flux spectral atlas of the Sun (Livingston \& Wallace 1991; $R=\lambda / \delta \lambda=500,000)$. The two stars, with quite different parameters, complement each other in constraining the Ce II $g f$-values, as well as in defining the contributions from blending lines from various other species. In general, the Ce II lines identified here are extremely weak, or undetectable, in the solar flux spectrum, while they are of moderate strength in the somewhat metal-poor K-giant $\alpha$ Boo and easily detectable, even in the lower-resolution APOGEE spectra of typical red giants. The most serious potentially blending lines in some of the Ce II lines are either high-excitation $(\chi \sim 5-10 \mathrm{eV})$ neutral lines of abundant species, such as Fe I, Si I, or C I that are weak in both the Sun and in $\alpha$ Boo, or molecular lines of $\mathrm{CO}, \mathrm{OH}$, or 

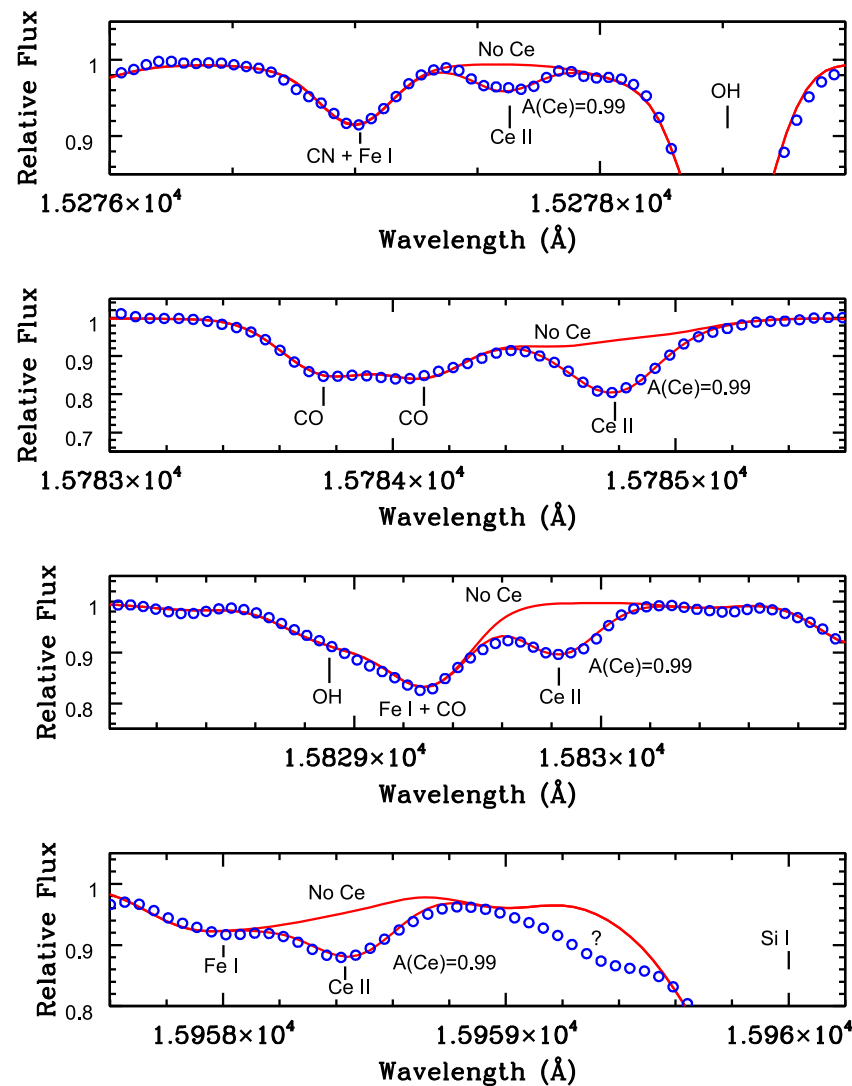

Figure 1. Four of the sample Ce II lines are shown in the observed FTS spectra of $\alpha$ Boo (open circles) and corresponding syntheses (solid curves). Each window contains one Ce II line, as indicated, with other prominent lines also labeled. The syntheses are shown for the cerium abundance derived from optical Ce II lines $(A(\mathrm{Ce})=0.99)$, which was derived using laboratory $g f$-values. Syntheses with no cerium are also shown. The derived Ce II abundances from optical lines were used to set the astrophysical $g f$-values for the H-band lines.

$\mathrm{CN}$. The detection of high-excitation, weak neutral lines in the Sun allows for estimates of their solar astrophysical $g f$-values, which then allows for the Ce II lines to be modeled; in all cases, these lines are quite weak in $\alpha$ Boo (much weaker than the Ce II lines).

If the blending lines are $\mathrm{CO}, \mathrm{OH}$, or $\mathrm{CN}$, their $g f$-values are not changed in the APOGEE line list, as these are welldetermined from the various references for these molecular lines (see Shetrone et al. 2015), and their strengths in $\alpha$ Boo are well accounted for in fitting the Ce II lines.

Figures 1 and 2 illustrate the Ce II lines observed in $\alpha$ Boo, along with synthetic fits to the spectrum. Each panel displays a small spectral region around a Ce II line, with two synthetic spectra overplotted on the observed $\alpha$ Boo spectrum: one synthesis with no cerium and one with the Ce II $g f$-value adjusted to fit $\alpha$ Boo with $A(\mathrm{Ce})=0.99$, as set by the optical $\mathrm{Ce}$ II analysis. The synthetic spectrum with no cerium provides an indication of how much contamination falls within the Ce II line. The derived $\log g f$-values are listed in Table 1. Details concerning each of the APOGEE Ce II lines are discussed below.

The $\lambda 15277 \AA$ Line: This Ce II line is found to be best fit in $\alpha$ Boo with an air wavelength of $15277.610 \AA$, compared to the value of $15277.65 \AA$ from Corliss (1973). This line is clean in $\alpha$ Boo and well separated from an $\mathrm{Fe} \mathrm{I} /{ }^{12} \mathrm{C}^{14} \mathrm{~N}$ blend
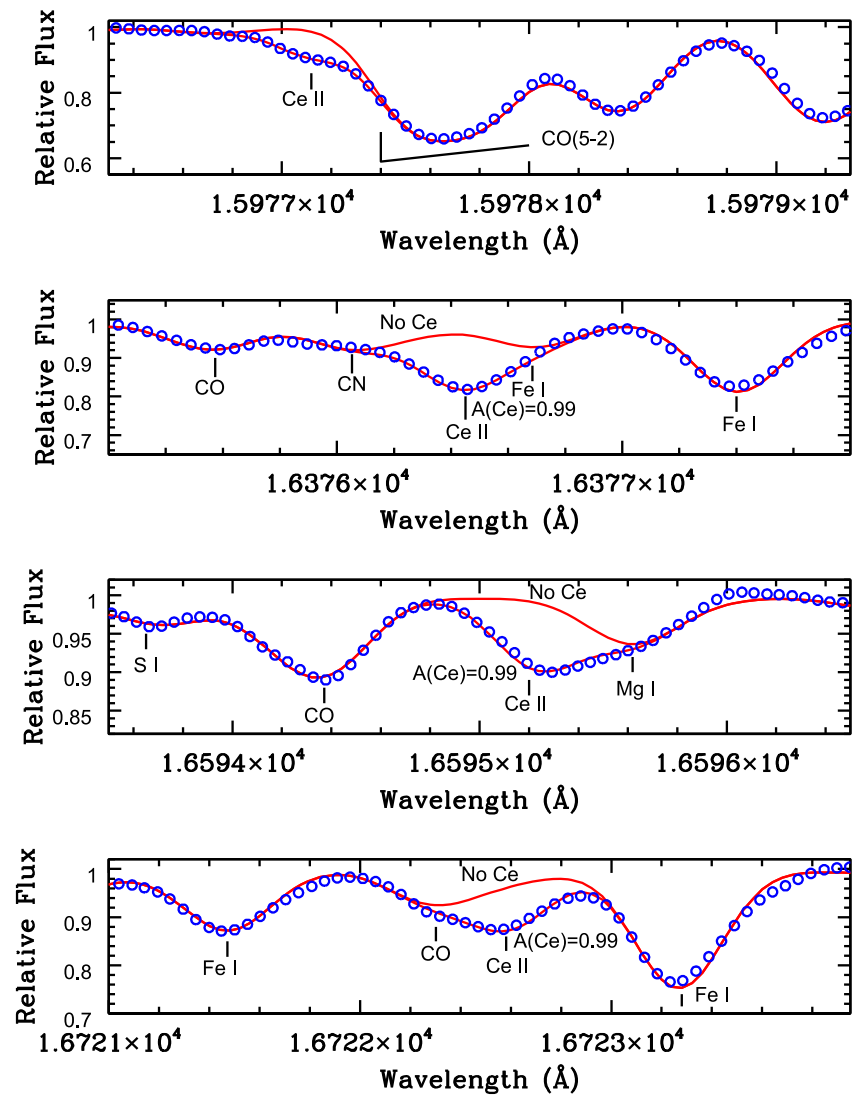

Figure 2. Four other sample Ce II lines shown in the observed FTS spectra of $\alpha$ Boo. Same as Figure 1.

to the blue and a strong ${ }^{16} \mathrm{OH}$ line to the red. Based on a cerium abundance of $A(\mathrm{Ce})=0.99$ in $\alpha$ Boo, this line has $\log g f=-1.94$. The line is extremely weak in the Sun, but detected, and the $\alpha$ Boo $g f$-value provides a fit to the Sun that is consistent with $\mathrm{A}(\mathrm{Ce})_{\odot}=1.58$ (Grevesse et al. 2015).

The $\lambda 15784 \AA$ Line: (see the upper panel of Figures 1-3) This Ce II line is a good example of the importance of using both the Sun and $\alpha$ Boo to constrain and set the astrophysical $g f$-value. Two C I lines straddle the wavelength of the Ce II line, with C I at $\lambda 15784.546 \AA$ and $\lambda 15784.920 \AA$, while Ce II is best fit with $\lambda 15784.786 \AA$ in $\alpha$ Boo (compared to the Corliss value of $\lambda 15784.75 \AA$ ). The $\mathrm{C}$ I lines have NIST $g f$-values of $\log g f=-0.592$ for the blue line and $\log g f=-0.729$ for the red line, with both lines having $\chi=9.631 \mathrm{eV}$ : these $g f$-values have grade-C accuracies, which indicate an uncertainty of $\pm 25 \%$. In $\alpha$ Boo, these C I lines are predicted to be very weak, but in the Sun are found to dominate, by far, any underlying Ce II absorption (although the solar C I lines are still weak, with depths of $\sim 5 \%-7 \%$, given the NIST $g f$-values). In fitting the Sun, it was found that the APOGEE line list for DR13 produced C I lines that were much too strong, with the APOGEE $g f$-values being $\log g f=-0.312$ for the blue line and -0.449 for the red line: these are larger than the NIST values by $+0.280 \mathrm{dex}$ for both lines. This difference arises because APOGEE attempts to fit the feature in $\alpha$ Boo without the Ce II line in the line list, with the Ce II line in $\alpha$ Boo being much stronger than $\mathrm{C} I$ in the Sun, and APOGEE giving a higher weight in attempting to fit this stronger feature in $\alpha$ Boo. The software pushes the NIST values to more closely match the relatively strong absorption in $\alpha$ Boo. 

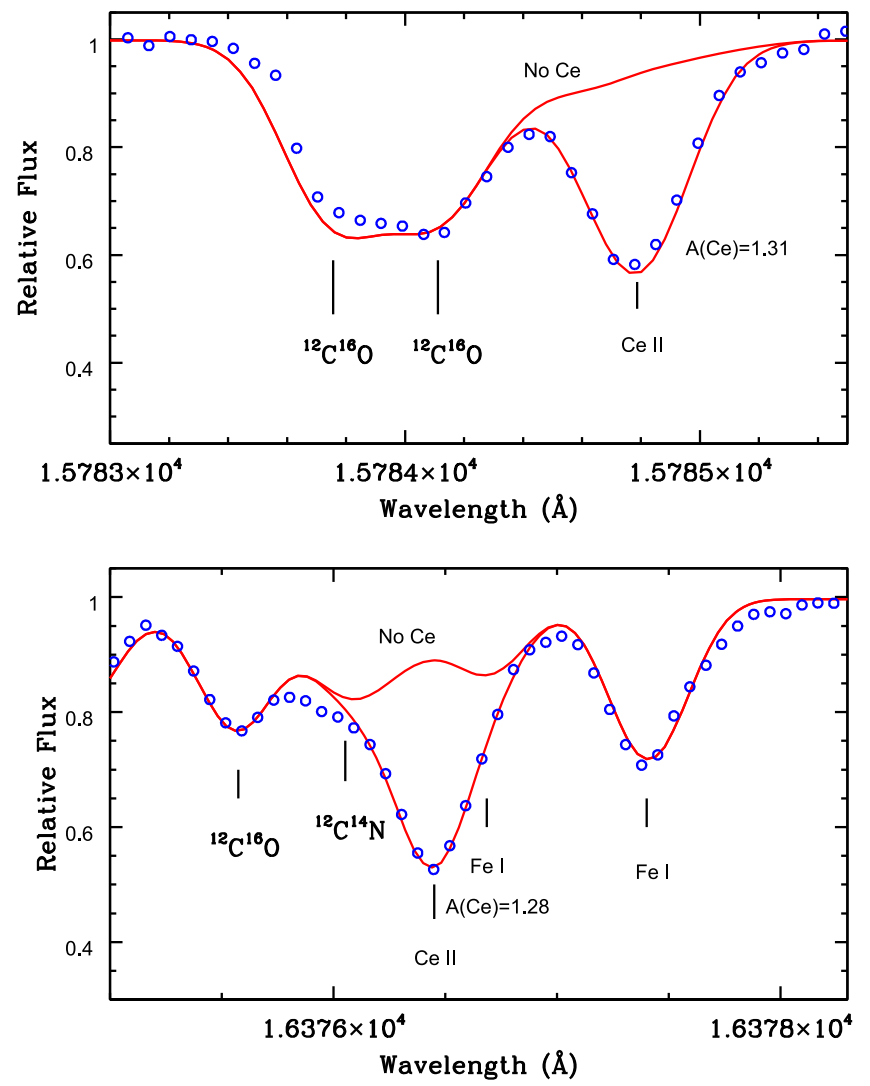

Figure 3. Two of the sample Ce II lines shown in the FTS spectrum of the M-giant $\beta$ And. Although cooler and more metal-rich than $\alpha$ Boo, the blending lines, relative to the $\mathrm{Ce}$ II lines, are not significantly worse.

In an iterative process, the NIST values of C I were used, along with the inclusion of the now known $\mathrm{Ce}$ II line to fit $\alpha$ Boo, and an initial $g f$-value was derived. The carbon abundance was set to the value derived by Smith et al. (2013) from CO lines using the APOGEE line list. The resulting Ce II $g f$-value was then used in modeling the solar spectrum, where it was found that the predicted Ce II line strength was insignificant compared to the two C I lines. Solar C I $g f$-values were then derived from the Sun, and these were found to be very close to the NIST values, with the solar $g f$-values being $\log g f=-0.572$ for the blue C I line (compared to the NIST value of -0.592 ) and -0.820 for the red line (compared to the NIST value of -0.729$)$. With these values of $\log g f$ for $\mathrm{C} \mathrm{I}$, consistent fits were found for both $\alpha$ Boo (with $A(\mathrm{Ce})=0.99$ ) and the Sun (for $A(\mathrm{Ce})=1.58$ ), with $\log g f(\mathrm{Ce}$ II $)=-1.54$.

The $\lambda 15958 \AA$ Line: In $\alpha$ Boo, this line was found to require a small wavelength adjustment of $-0.01 \AA$ from the value in Corliss (1973), so the observed line has $\lambda=15958.390 \AA$. This is a rather crowded spectral region, with a high-excitation Fe I line very close by at $\lambda=15958.168 \AA(0.222 \AA$ to the blue) and a weak ${ }^{12} \mathrm{C}^{16} \mathrm{O}$ line some $+0.058 \AA$ to the red. A more distant ${ }^{12} \mathrm{C}^{14} \mathrm{~N}$ line lies $-0.511 \AA$ to the blue and blends slightly with $\mathrm{Ce}$ II in $\alpha$ Boo. In $\alpha$ Boo, the region containing the $\mathrm{CN}, \mathrm{Fe}$, $\mathrm{CO}$, and $\mathrm{Ce}$ II lines is dominated by $\mathrm{Ce}$ II absorption, while in the Sun, neither Ce II, nor CO and CN are detected, while Fe I is detected as a weak line. Due to the lack of Ce II line in the older APOGEE line list, the attempt to account for the absorption observed in $\alpha$ Boo in this small wavelength region led to an $\mathrm{Fe}$ I $g f$-value that yields a theoretical solar $\mathrm{Fe} \mathrm{I}$ line that is much too strong.
Using the Sun to set the Fe I $\lambda 15958.168 \AA g$-value yields $\log g f=-2.65$ for this line. Using this Fe I oscillator strength to synthesize $\alpha$ Boo results in $\log g f=-1.71$ for the Ce II line. This value is consistent with the non-detection of the Ce II line in the Sun. The blended feature is now fit very well in $\alpha$ Boo.

The $\lambda 15977 \AA$ Line: The initial reaction was that the Ce II line at $\lambda 15977.12 \AA$ would not be useful, due to its proximity to and blending with the nearby ${ }^{12} \mathrm{C}^{16} \mathrm{O}(5-3)$ bandhead in $\alpha$ Boo. It turns out, however, that there is obvious, significant missing absorption at the blue edge of the $\mathrm{CO}$ bandhead, coinciding with the wavelength of the Ce II line and at the approximate strength expected, given the other Ce II lines in the APOGEE spectral window. The $\mathrm{CO}$ lines are well fit in $\alpha$ Boo and fitting the Ce II line requires $\log g f=-2.10$, with no wavelength shift ( $\lambda 15977.12 \AA$ from Corliss 1973). In addition, at the red edge of the line, there is some contribution from a $\mathrm{Nd}$ II line at $\lambda 15978.016 \AA$ (Hasselquist et al. 2016).

The CO lines in the Sun are very weak, but at about the same strength as the Ce II line. The $g f$-value derived from $\alpha$ Boo results in a good fit to the solar Ce II line with solar cerium abundance.

The $\lambda 16327 \AA$ Line: The Ce II line at $\lambda 16327.32 \AA$ is not the dominant absorber in this region of the $\alpha$ Boo spectrum, but is found to be a useful abundance indicator in the metal-poor stars analyzed here. In $\alpha$ Boo and the other normal-abundance red giants, this absorption feature is dominated by a ${ }^{13} \mathrm{C}^{16} \mathrm{O}$ line with $\chi=0.882 \mathrm{eV}$. The astrophysical $g f$-value derived here was set using the extreme s-process enriched star 2M16011638-1201525, and this line is useful in abundance analyses of metal-poor stars, especially the warmer giants, since the $\mathrm{CO}$ absorption decreases as the square of decreasing metallicity, as well as weakening with increasing $T_{\text {eff }}$.

The $\lambda 16376 \AA$ Line: (see the lower panel of Figures 1-3) The wavelength of this Ce II line had to be adjusted slightly, by $+0.020 \AA$ from the Corliss (1973) value to $\lambda 16376.460 \AA$. There is an underlying Fe I line, with $\chi=6.365 \mathrm{eV}$, at $\lambda 16376.686 \AA$ and because Ce II was not included in the determination of APOGEE astrophysical $g f$-values, the $g f$-value for this line (which has no NIST value) was overestimated. The Fe I line is visible in the Sun and is best fit with $\log g f=-1.522$. Using this value for the oscillator strength of $\mathrm{Fe}$ I, the Ce II line in $\alpha$ Boo requires $\log g f=-1.790$. There is a weak ${ }^{12} \mathrm{C}^{16} \mathrm{O}$ line and a weak ${ }^{12} \mathrm{C}^{14} \mathrm{~N}$ line partially blending the Ce II line to the blue.

The $\lambda 16595 \AA$ Line: This Ce II line at $\lambda 16595.18 \AA$ is blended with $\mathrm{MgI}$ at $\lambda 16595.620 \AA$, which has $\chi=6.516$ $\mathrm{eV}$ and an astrophysical $g f$-value that is very close to its value in the NIST database. Although blended with $\mathrm{Mg}$ I, the nearby regions are fairly clean and the $\mathrm{Ce}$ II absorption remains well defined in the red giants. The Ce II oscillator strength derived from the $\alpha$ Boo spectrum is $\log g f=-2.19$, with $\lambda 16595.233 \AA$; this line may be the most useful of the Ce II lines in the APOGEE window, as it is detectable across a broad range of stellar parameters, even with the blending $\mathrm{Mg}$ I line.

The $\lambda 16722 \mathrm{~A}$ Line: Spectral fitting required a wavelength shift of $+0.03 \AA$ for this line relative to Corliss (1973), to $\lambda 16722.600 \AA$. As is the case for a few of the other Ce II lines, this line has a weak, high-excitation, neutral line very close, which is $\mathrm{Si}$ I at $\lambda 16722.704 \AA$ and having $\chi=7.029 \mathrm{eV}$. The astrophysical $g f$-value derived in the DR13 and earlier versions of the APOGEE line list did not account for the Ce II absorption, so the Si I $g f$-value has been overestimated. The 
Table 3

Uncertainties in $\log g f$

\begin{tabular}{|c|c|c|c|c|c|c|}
\hline $\begin{array}{l}\text { Ce II } \\
\lambda_{\text {Air }}(\AA)\end{array}$ & $\begin{array}{c}\Delta T_{\text {eff }} \\
(+30 \mathrm{~K})\end{array}$ & $\begin{array}{c}\Delta \log g \\
(+0.05 \\
\operatorname{dex})\end{array}$ & $\begin{array}{c}\Delta \\
{[\mathrm{M} / \mathrm{H}]} \\
(+0.05 \\
\operatorname{dex})\end{array}$ & $\begin{array}{c}\Delta \xi \\
(+0.05 \\
\left.\mathrm{km} \mathrm{s}^{-1}\right)\end{array}$ & $\begin{array}{l}\Delta \text { Cont } \\
(+0.01)\end{array}$ & $\Delta_{\mathrm{T}}$ \\
\hline 15277 & +0.07 & +0.08 & +0.04 & 0.00 & +0.01 & 0.11 \\
\hline 15784 & +0.07 & +0.08 & +0.04 & 0.00 & +0.01 & 0.11 \\
\hline 15829 & +0.06 & +0.07 & +0.03 & -0.01 & 0.00 & 0.10 \\
\hline 15958 & +0.07 & +0.08 & +0.04 & 0.00 & +0.01 & 0.11 \\
\hline 15977 & +0.06 & +0.07 & +0.03 & 0.00 & 0.00 & 0.10 \\
\hline $16327^{\mathrm{a}}$ & +0.02 & +0.06 & +0.01 & -0.01 & +0.01 & 0.07 \\
\hline 16376 & +0.07 & +0.07 & +0.03 & 0.00 & 0.00 & 0.10 \\
\hline 16595 & +0.07 & +0.07 & +0.03 & 0.00 & +0.01 & 0.10 \\
\hline 16722 & +0.06 & +0.07 & +0.03 & -0.01 & 0.00 & 0.09 \\
\hline
\end{tabular}

Note.

${ }^{a}$ The $g f$-value for this line was derived from 2M16011638-1201525 and uncertainties were calculated using $\Delta T_{\text {eff }}=+90 \mathrm{~K}, \Delta \log g=+0.2$, $\Delta[\mathrm{M} / \mathrm{H}]=+0.10$, and $\Delta \xi=+0.25 \mathrm{~km} \mathrm{~s}^{-1}$

Si I line in the Sun is best fit with $\log g f=-2.40$. Using this value in the analysis of $\alpha$ Boo results in $\log g f=-1.65$ for this Ce II line.

\subsection{Uncertainties in the Astrophysical gf-Values}

Uncertainties in the astrophysical $g f$-values derived in the previous section can be evaluated by considering how well the stellar parameters are known for $\alpha$ Boo. In addition, as in all quantitative stellar spectroscopic analyses, there are limitations inherent in the analysis technique: here, that technique is a $1 \mathrm{D}$ static model atmosphere LTE analysis. As noted previously, this is the same type of analysis employed by the APOGEE/ASPCAP machinery, and its use here helps ensure that the $g f$-values for Ce II are defined on the APOGEE abundance scale.

Estimates of the uncertainties in the Ce II $g f$-values are presented in Table 3; these were derived by varying the $\alpha$ Boo parameters by the uncertainties in the parameters as estimated in Ramirez \& Allende Prieto (2011): $T_{\text {eff }}=4286 \pm 30 \mathrm{~K}$, $\log g=1.66 \pm 0.05$, and $[\mathrm{Fe} / \mathrm{H}]=-0.52 \pm 0.04$. Forcing the Ce II lines to yield a constant abundance $(A(\mathrm{Ce})=0.99)$ with altered $\alpha$ Boo parameters results in changes in $\log g f$ for each line as listed in Table 3 . The changes in $\log g f$ required for each change in the particular stellar parameter were summed in quadrature to produce the total uncertainty in $\log g f\left(\Delta_{\text {total }}\right.$ presented in Table 3; this is an upper limit to the uncertainty as some of these errors may cancel out with the covariances between the parameters.) The behaviors of each of the Ce II lines as a function of each stellar parameter are similar and the values of $\Delta_{\text {total }}$ are about the same, at $\sim 0.1$ dex. Uncertainties in any of the $\alpha$ Boo parameters would thus produce an almost constant offset in derived cerium abundances in other samples of stars. The uncertainty in the absolute $\alpha$ Boo cerium abundance $( \pm 0.05$ dex $)$ would affect each of the APOGEE Ce II lines equally, again leading to an offset in derived cerium abundances. With nine lines and a precision of 0.1 dex for each line, the APOGEE spectral window has the potential of providing a precision in the derived cerium abundances of about 0.03 dex.

The overall consistency of the astrophysical $g f$-values derived here, which are tied ultimately to the parameters of $\alpha$
Boo, can be tested using the target star 2M16011638-1201525, as its low metallicity but extreme s-process enrichment provides a set of clean, well-defined Ce II lines. As previously mentioned, 2M16011638-1201525 was also used to set the astrophysical $g f$-value for the weakest Ce II line at $\lambda 16327.32 \AA$ since this line was dominated by other blending species in $\alpha$ Boo. Therefore, the $\mathrm{Ce}$ abundance derived for this star was that derived from the seven other Ce II lines: $A(\mathrm{Ce})=1.30 \pm 0.06$ (Table 4). The standard deviation of the mean is just slightly larger than the one obtained for $\alpha$ Boo and the mean cerium abundance is also in agreement with the cerium abundance derived from the optical lines discussed in Section 2.2: $A(\mathrm{Ce})_{\text {optical }}=1.22 \pm 0.06$; the $0.08 \mathrm{dex}$ offset found between the two sets of abundances is in line with the uncertainties estimated in Table 3.

\section{Cerium Abundances in Selected Samples of Red Giants}

The usefulness of the Ce II lines identified in the APOGEE region was tested via abundance analyses of cerium in a small sample of selected field and open cluster red giants. The sample stars analyzed and their stellar parameters and cerium abundances are presented in Table 4, while the individual line abundances are presented in Table 5.

\subsection{Bright Field Red Giants with FTS Spectra}

The analysis of the Ce II lines in the FTS spectra of $\delta$ Oph, $\beta$ And, $\mu$ Leo, and HD 199799 (Section 2.1) was carried out using the same stellar parameters as in Smith et al. (2013; Table 3). The Ce II lines in the M-giants, $\delta$ Oph and $\beta$ And, are well defined; sample fits to their FTS spectra are shown in Figure 3. In the spectra of such red giants, $\mathrm{CO}$ or $\mathrm{OH}$ lines tend to be the blending species to $\mathrm{Ce}$ II; however, since there are large numbers of other $\mathrm{CO}$ and $\mathrm{OH}$ lines which set, respectively, the carbon and oxygen abundances, such blends are well-constrained and do not seriously compromise the Ce II lines. The individual line abundances in these stars (from seven Ce II lines) agree quite well, and the standard deviation of the $\mathrm{Ce}$ abundances is quite small: $\sim \pm 0.03-0.04$ dex. Their values of $[\mathrm{Ce} / \mathrm{Fe}]$ are near solar-scaled, with both being near $[\mathrm{Ce} / \mathrm{Fe}] \sim 0.0$, which is to be expected for nearsolar-metallicity disk giants.

The metal-rich K-giant $\mu$ Leo presents a different type of spectrum compared to M-giants, due to its hotter $T_{\text {eff }}$ and significantly higher metallicity; here the typical blending species to $\mathrm{Ce}$ II tends to be $\mathrm{CN}$. The $\mathrm{CN}$ lines provide a more general weak haze of background blending features across the APOGEE window, while the Ce II lines themselves are somewhat weaker in this higher-gravity giant. The value of $[\mathrm{Ce} / \mathrm{Fe}]=-0.18$ is mildly subsolar and in agreement with literature values for stars with similar metallicities in the solar neighborhood (Section 4.1).

The Ce II lines are noticeably strengthened in the s-process rich star HD 199799, for which we derive a cerium abundance of $A(\mathrm{Ce})=1.74 \pm 0.05([\mathrm{Ce} / \mathrm{Fe}]=+0.36)$. This represents a modest enhancement of the s-process and is similar to the enhancement of Nd found by Smith \& Lambert (1990) for this star. The Ce II lines $\lambda 15784$ and $\lambda 16376$ are shown for $\alpha$ Boo in Figures 1 and 2, respectively. These Ce II lines are also shown in Figure 3 for $\beta$ And, and in Figure 4 for HD 199799, 
Table 4

Stellar Parameters and Abundances

\begin{tabular}{|c|c|c|c|c|c|c|}
\hline Star & $T_{\text {eff }}(\mathrm{K})$ & $\log g\left(\mathrm{~cm} \mathrm{~s}^{-2}\right)$ & $\xi\left(\mathrm{km} \mathrm{s}^{-1}\right)$ & $A(\mathrm{Fe})$ & $A(\mathrm{Ce})$ & {$[\mathrm{Ce} / \mathrm{Fe}]$} \\
\hline \multicolumn{7}{|l|}{ "Standard" Stars } \\
\hline Sun & 5777 & 4.438 & 1.0 & 7.45 & $1.58(0.03)$ & 0.00 \\
\hline$\alpha$ Boo & 4286 & 1.66 & 1.8 & 6.98 & $0.99(0.05)^{\mathrm{a}}$ & -0.12 \\
\hline$\beta$ And & 3825 & 0.9 & 2.2 & 7.23 & $1.32(0.03)$ & -0.04 \\
\hline$\delta \mathrm{Oph}$ & 3850 & 1.2 & 1.9 & 7.44 & $1.57(0.04)$ & 0.00 \\
\hline$\mu$ Leo & 4550 & 2.1 & 1.8 & 7.76 & $1.71(0.06)$ & -0.18 \\
\hline HD 199799 & 3400 & 0.5 & 2.4 & 7.25 & $1.74(0.05)$ & 0.36 \\
\hline \multicolumn{7}{|l|}{ APOGEE Field Stars } \\
\hline $2 \mathrm{M} 07182377+0720134$ & 4200 & 1.68 & 1.64 & 6.98 & $1.13(0.05)$ & 0.02 \\
\hline 2M15203732-0207263 & 3986 & 1.20 & 1.67 & 6.47 & $0.81(0.05)$ & 0.21 \\
\hline HD 35155 & 3650 & 0.8 & 2.5 & 6.94 & $2.39(0.15)$ & 1.32 \\
\hline \multicolumn{7}{|l|}{ APOGEE NGC 6819 Stars } \\
\hline $2 \mathrm{M} 19404965+4014313$ & 4710 & 2.56 & 1.43 & 7.50 & $1.55(0.06)$ & -0.08 \\
\hline $2 \mathrm{M} 19411367+4003382$ & 4688 & 2.44 & 1.43 & 7.46 & $1.53(0.06)$ & -0.07 \\
\hline \multicolumn{7}{|l|}{ APOGEE N-rich Stars } \\
\hline 2M16011638-1201525 & 4305 & 0.60 & 1.65 & $6.06(0.06)$ & $1.30(0.06)$ & 1.11 \\
\hline 2M17205201-2903061 & 3998 & 0.73 & 2.04 & $6.60(0.06)$ & $1.01(0.11)$ & 0.28 \\
\hline 2M17333623-2548156 & 4092 & 0.73 & 2.04 & $6.41(0.08)$ & $0.81(0.05)$ & 0.27 \\
\hline 2M17334208-2958347 & 3975 & 0.51 & 2.24 & $6.47(0.03)$ & $0.98(0.06)$ & 0.38 \\
\hline 2M18032356-3001588 & 3990 & 0.53 & 2.22 & $6.31(0.06)$ & $0.54(0.04)$ & 0.10 \\
\hline 2M18334592-2903253 & 4161 & 0.90 & 1.89 & $6.64(0.04)$ & $0.99(0.09)$ & 0.22 \\
\hline 2M18442352-3029411 & 3996 & 0.73 & 2.04 & $6.64(0.06)$ & $1.20(0.11)$ & 0.43 \\
\hline
\end{tabular}

Note.

${ }^{a}$ From optical lines with Lawler et al. (2009) $g f$-values

illustrating the behavior of the nearby blending lines as $T_{\mathrm{eff}}$ decreases.

\subsection{Field and Open Cluster Red Giants Observed by APOGEE}

To further characterize the Ce II lines and to test their utility in the lower-resolution APOGEE spectra, a few red giants from the APOGEE/ASPCAP DR13 database were analyzed for their $\mathrm{Ce}$ abundances. These stars include two members of the solarmetallicity open cluster NGC 6819, with both stars having $T_{\text {eff }} \sim 4700 \mathrm{~K}$ and falling near the red clump in the colormagnitude diagram. Two moderately metal-poor field red giants were also chosen, with effective temperatures of 4200 and $3990 \mathrm{~K}$, and $[\mathrm{Fe} / \mathrm{H}]=-0.52$ and -0.98 , respectively. In addition, the extremely s-process enhanced S-star, HD 35155 (Smith \& Lambert 1990) was studied. The stellar parameters and metallicities for these stars are from DR13. Figure 5 shows three of the Ce II lines in the NGC 6819 member 2M19411367 +4003382 , which is one of the hotter red giants included here. The Ce II lines are clearly detected and modeled. Figure 6 illustrates the same three Ce II lines in the moderately metalpoor field red giant 2M15203732-0207263; again, the Ce II lines are clearly detected and well fit.

Our results indicate that in the non-s-process enriched giants, the $[\mathrm{Ce} / \mathrm{Fe}]$ values are unremarkable, with $[\mathrm{Ce} / \mathrm{Fe}] \sim-0.05$ to +0.20 ; the value of +0.20 is found in the most metal-poor $([\mathrm{Fe} / \mathrm{H}]=-1.0)$ star, and this slightly positive value may reflect Galactic chemical evolution, to be discussed in Section 4.1. The S-star HD 35155 exhibits very strong absorption at all of the Ce II lines and demonstrates the extreme s-process rich nature of this star. The derived abundance of $A(\mathrm{Ce})=2.39 \pm 0.15$ and $[\mathrm{Fe} / \mathrm{H}]=-0.51$ yields $[\mathrm{Ce} / \mathrm{Fe}]=+1.32$, a significant s-process enhancement (see also Hasselquist et al. 2016).

\subsection{Very Nitrogen-rich Metal-poor Red Giants from the APOGEE Sample}

A sample of seven metal-poor stars, which exhibit the chemical peculiarities of being $\mathrm{N}$ and $\mathrm{Al}$ rich, were included here in order to probe their $\mathrm{Ce}$ abundances and to see whether s-process enhancements go hand in hand with the enhanced $\mathrm{N}$ and Al. These red giants have metallicities that range from $[\mathrm{Fe} / \mathrm{H}] \sim-1.4$ to -0.8 , and effective temperatures between $T_{\text {eff }} \sim 4000-4300 \mathrm{~K}$. Five of these chemically peculiar red giants with spectra with high signal-to-noise ratio $(\mathrm{S} / \mathrm{N})$ were taken at random from Schiavon et al. (2017a); their paper argues that the population of $\mathrm{N}$ - and Al-rich stars identified by them in the APOGEE database may be stripped or escaped members of globular clusters. One star was taken from Fernández-Trincado et al. (2016), 2M16011638-1201525; both Fernández-Trincado et al. (2016) and Pereira et al. (2017) suggest that 2M16011638-1201525 might have been ejected from the peculiar globular cluster/dwarf galaxy remnant $\omega$ Cen. Finally, one target (2M18032356-3001588; Arp IV-203) is a member of the bulge globular cluster NGC 6522 (Schiavon et al. 2017b) and was previously analyzed in the bulge studies by Ryde et al. (2010) and Fulbright et al. (2007).

The effective temperatures for all of these stars (except 2M16011638-1201525; Section 3.2) are from DR13, while their $\log g$ values were derived using the PARSEC isochrones for an age of $8 \mathrm{Gyr}$ (Bressan et al. 2012); the DR13 $\log g$ values for these stars show offsets due to their peculiar abundances and are not used here. We also derived their $\mathrm{Fe}$ abundances manually based on individual Fe I lines in a manner similar to that presented in Cunha et al. (2015; Table 3).

As expected from the strength of the Ce II lines in the spectrum of 2M16011638-1201525, this very N- and Al-rich star shows an extreme enhancement in the cerium abundance of $[\mathrm{Ce} / \mathrm{Fe}]=+1.11$. Five of the other $\mathrm{N}$ - and Al-rich stars 
Table 5

Ce II Line-by-Line Abundances

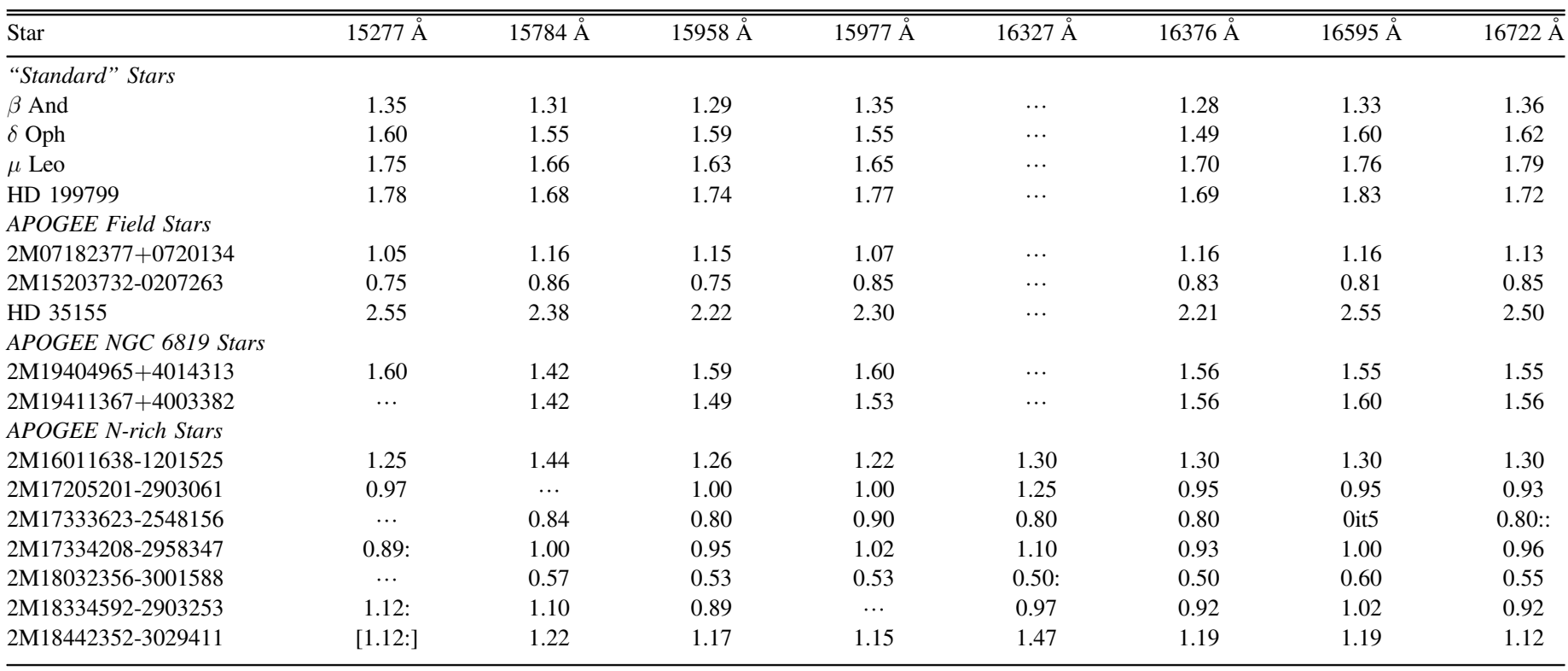
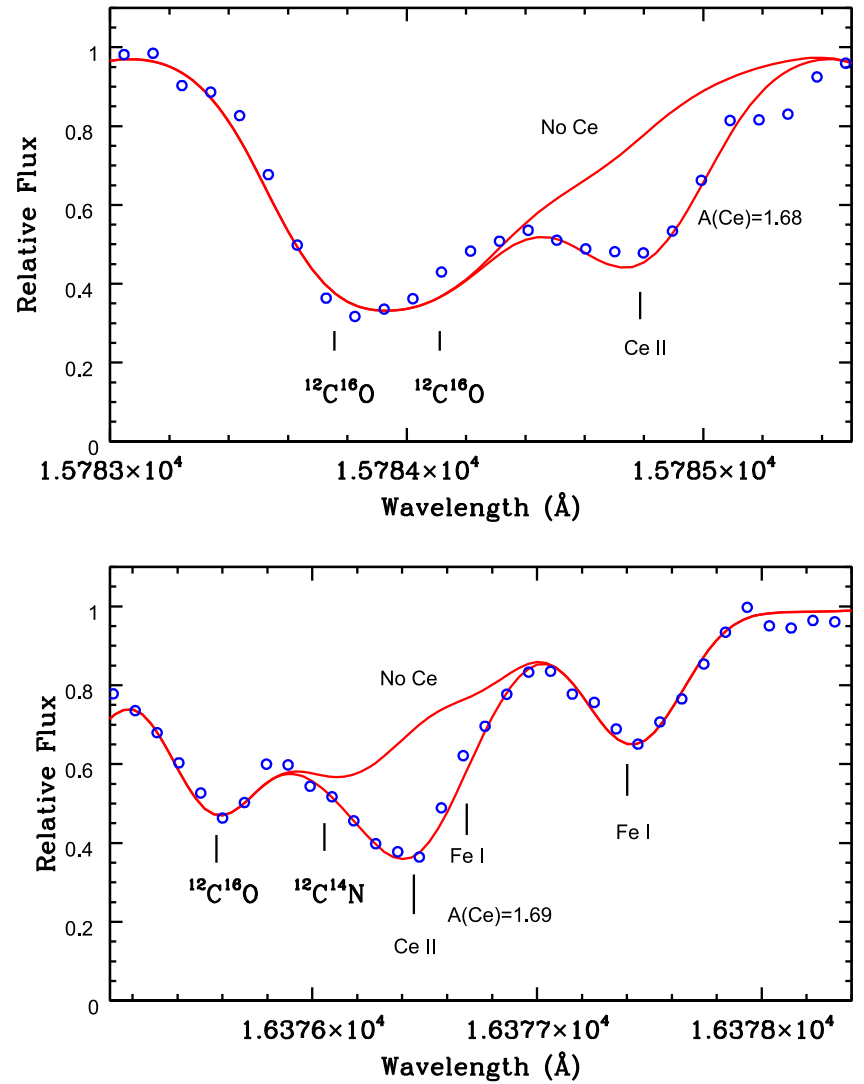

Figure 4. The two Ce II lines from Figure 3 are now shown in the FTS spectrum of the TP-AGB star HD 199799, which is much cooler and has a lower gravity than either $\alpha$ Boo or $\beta$ And. Cerium in this third dredge-up giant is mildly enhanced and the blending with $\mathrm{CO}$ has increased, but the model fit to the observed spectrum remains good.

analyzed do not show such extreme behavior and were found to have mildly enhanced values of $[\mathrm{Ce} / \mathrm{Fe}] \sim+0.2-+0.4$, while the bulge globular cluster member 2M18032356-3001588 has a slightly lower value of $[\mathrm{Ce} / \mathrm{Fe}]+0.1$. In this small sample, the
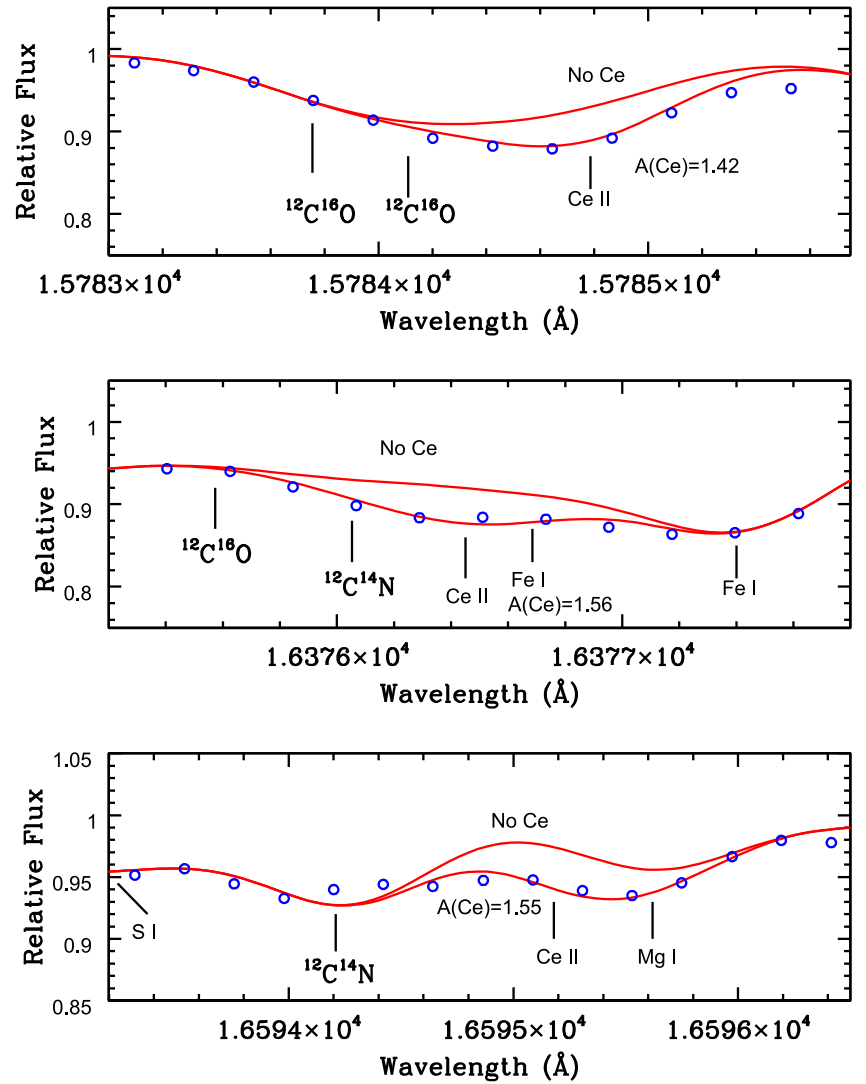

Figure 5. Three of the Ce II lines in the APOGEE spectrum of the NGC 6819 red giant $2 \mathrm{M} 19411367+4003382$, which is on the lower RGB, with $T_{\text {eff }}=4695 \mathrm{~K}, \log g=2.45$, and $[\mathrm{Fe} / \mathrm{H}]=+0.01$. Each panel shows one Ce II line and identifies other nearby lines, with the observed spectra shown as open circles and sample synthetic spectra plotted as solid curves. Two synthetic spectra are shown in each panel, with one synthesis having no cerium and the other having the best-fit $\mathrm{Ce}$ abundance for that line.

significant enhancements of both $\mathrm{N}$ and $\mathrm{Al}$ do not correlate with extreme increases in the $\mathrm{Ce}$ abundance, except for one star. 

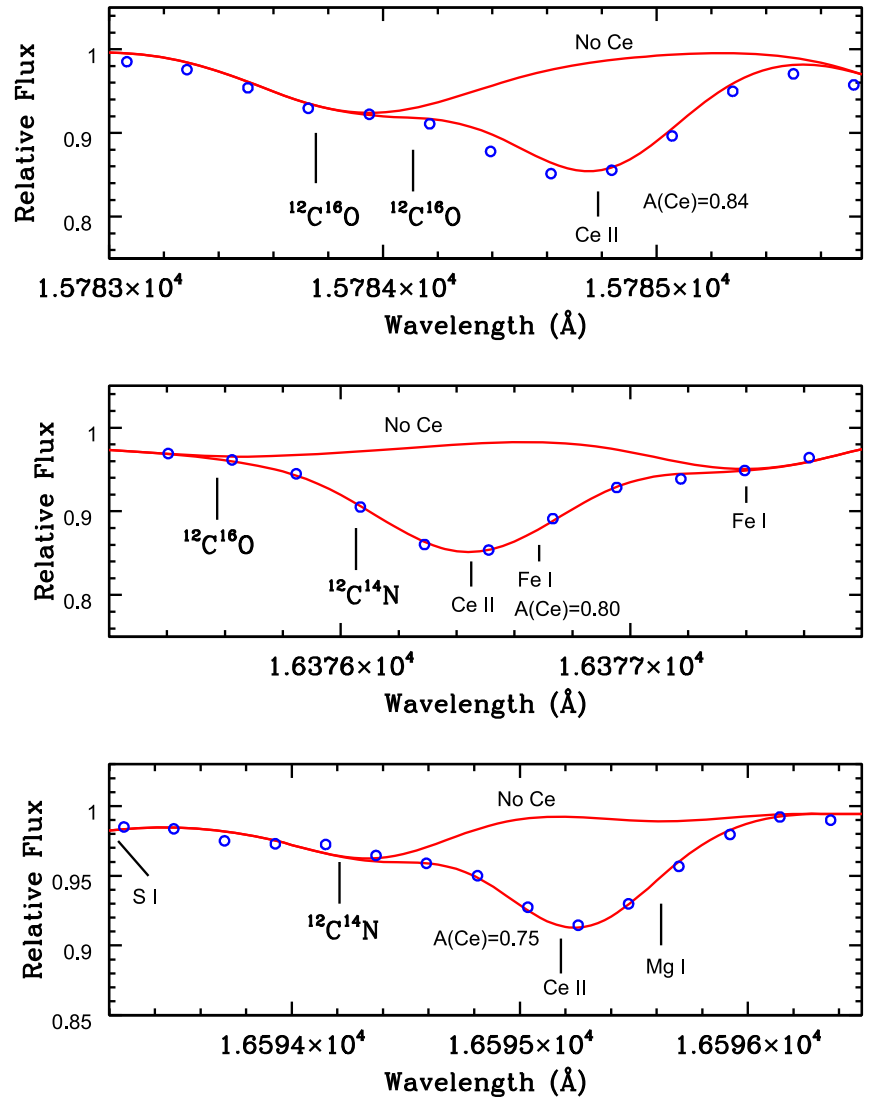

Figure 6. Three of the Ce II lines in the APOGEE spectra of the cool, moderately metal-poor field red giant $2 \mathrm{M} 15203732-0207263\left(T_{\text {eff }}=3985 \mathrm{~K}\right.$, $\log g=1.20,[\mathrm{Fe} / \mathrm{H}]=-0.98)$ : symbols are the same as in Figure 2. Note that even in the cooler, but lower gravity red giants, the Ce II lines remain well defined at moderately low metallicities.

\subsection{Abundance Sensitivities and Uncertainties}

To compute the sensitivities of the derived cerium abundances to the uncertainties in the adopted stellar parameters, we used two model atmospheres as baselines: $T_{\text {eff }}=3850 \mathrm{~K}, \log g=1.20$, $\xi=1.9 \mathrm{~km} \mathrm{~s}^{-1}$, and $[\mathrm{Fe} / \mathrm{H}]=0.0$ (corresponding to $\delta \mathrm{Oph}$ ), and $T_{\text {eff }}=3990 \mathrm{~K}, \log g=0.53, \xi=1.43 \mathrm{~km} \mathrm{~s}^{-1}$, and [Fe/ $\mathrm{H}]=-1.15$ (corresponding to 2M18032356-3001588, a star in NGC 6522). We then computed new model atmospheres by changing the stellar parameters over typical estimated uncertainties, $T_{\text {eff }}$ by $+90 \mathrm{~K}, \log g$ by $+0.2 \mathrm{dex}, \xi$ by $+0.25 \mathrm{~km} \mathrm{~s}^{-1}$, and metallicity by $+0.1 \mathrm{dex}$, one parameter at a time. The sensitivities of the derived Ce abundances are presented in Table 6; the last column in the table corresponds to the errors added in quadrature, which are upper limits to the uncertainties. The Ce II lines are, as expected for ionized species, most sensitive to the surface gravity values.

\section{Discussion}

The cerium abundances derived from the $\mathrm{Ce}$ II lines characterized and analyzed here for the select sample of red giants are compared to $\mathrm{Ce}$ abundances derived from optical Ce II lines by using the behavior of $[\mathrm{Ce} / \mathrm{Fe}]$ as a function of $[\mathrm{Fe} / \mathrm{H}]$ to investigate whether there are significant differences or obvious offsets. We also evaluate the strength and the associated error in fitting one of the strong Ce II lines across a range of effective temperatures, surface gravities, and metallicities to map detection limits of Ce II in red giants of various stellar parameters.

\section{1. $[\mathrm{Ce} / \mathrm{Fe}]$ versus $[\mathrm{Fe} / \mathrm{H}]$ from the APOGEE Ce II Lines}

The overall consistency in the Ce II astrophysical $g f$-values derived in this study can be tested by comparing the abundances derived here with cerium abundances from the literature. Figure 7 plots values of $[\mathrm{Ce} / \mathrm{Fe}]$ versus $[\mathrm{Fe} / \mathrm{H}]$ for stars analyzed in this study, along with samples of $F$ and $G$ dwarfs from Reddy et al. (2003, 2006; cyan open triangles and pentagons), Mishenina et al. (2013; green open squares), Battistini \& Bensby (2016; red asterisks), and Fishlock et al. (2017; small blue open squares) for values of $[\mathrm{Fe} / \mathrm{H}]$ covering the metallicity range of the majority of the Galactic thin and thick disks. The trends of $[\mathrm{Ce} / \mathrm{Fe}]$ with $[\mathrm{Fe} / \mathrm{H}]$, defined by the general field-star populations (Reddy et al. 2003; and Reddy et al. 2006; Mishenina et al. 2013; Battistini \& Bensby 2016; and Fishlock et al. 2017) are in overall agreement, although there is significant scatter in $[\mathrm{Ce} / \mathrm{Fe}]$. These reveal values of $[\mathrm{Ce} / \mathrm{Fe}]$ that are roughly solar $(\sim 0.0$, although with some scatter) from metallicity values between $[\mathrm{Fe} / \mathrm{H}] \sim-1.0$ and 0 . At metallicities above solar, from $[\mathrm{Fe} / \mathrm{H}]=0.0$ to +0.3 , $[\mathrm{Ce} / \mathrm{Fe}]$ declines slightly to -0.2 . The behavior of $[\mathrm{Ce} / \mathrm{Fe}]$ below $[\mathrm{Fe} / \mathrm{H}] \leqslant-1.0$ is less clear from the stellar samples shown in Figure 7, due to a small number of stars. When considering the results from all of the studies, there is no evidence of significant trends with decreasing $[\mathrm{Fe} / \mathrm{H}]$, but a scatter of $\sim \pm 0.25$ dex around $[\mathrm{Ce} / \mathrm{Fe}] \sim 0.0$.

Examining the behavior of $[\mathrm{Ce} / \mathrm{Fe}]$ as a function of metallicity for $[\mathrm{Fe} / \mathrm{H}] \sim-0.7$ and above (Figure 7), it is found that the "FTS standards" ( $\alpha$ Boo, $\beta$ And, $\delta$ Oph, and $\mu$ Leo; black filled squares with error bars) fall roughly in the center of the scatter defined by the optical analyses, as do the two members of the open cluster NGC 6819 (black filled circles with error bars), and the field red giant with $[\mathrm{Fe} / \mathrm{H}] \sim$ -0.5 that was selected from the APOGEE sample as having a typical red giant star composition (black filled pentagon). Note that the two known s-process enhanced red giants, HD 199799 and HD 35155 (represented by black filled triangles), are easily confirmed as being s-process enhanced via the infrared Ce II lines in the APOGEE window.

When examining the metallicity regime with $[\mathrm{Fe} / \mathrm{H}]<-0.7$, it should be noted that seven out of the eight stars analyzed here are $\mathrm{N}$ - and Al-rich red giants (black open squares) taken from Schiavon et al. (2017a, 2017b) and Fernández-Trincado et al. (2016); these stars likely represent a different stellar population, as discussed in Section 3.3. One of these stars, 2M16011638$1201525([\mathrm{Fe} / \mathrm{H}] \sim-1.4)$, is found here to be extremely $\mathrm{Ce}$ enriched. This extreme Ce enrichment is also confirmed from an independent analysis in the optical by Pereira et al. (2017), who obtained $[\mathrm{Ce} / \mathrm{Fe}]=+0.95 \pm 0.13$. Five of the other six $\mathrm{N}$ - and Al-rich stars fall on the upper part of the $[\mathrm{Ce} / \mathrm{Fe}]$ scatter at $[\mathrm{Fe} / \mathrm{H}] \sim-1.0$ (Figure 7 ), possibly indicating that this stellar population contains a significant fraction of s-process enhanced members. Curiously, the one star from the group of $\mathrm{N}$ - and $\mathrm{Al}-$ rich red giants with the lowest value of $[\mathrm{Ce} / \mathrm{Fe}]=+0.10$ is 2M18032356-3001588, a member of the bulge globular cluster NGC 6522.

Overall, the results shown in Figure 7 demonstrate that the Ce II $g f$-values derived in this study yield cerium abundances 
Table 6

Ce II Abundance Sensitivities to Stellar Parameter Uncertainties

\begin{tabular}{|c|c|c|c|c|c|}
\hline $\begin{array}{l}\text { Baseline Model } \\
T_{\text {eff }} ; \log g ;[\mathrm{Fe} / \mathrm{H}]\end{array}$ & $\begin{array}{c}\Delta T_{\text {eff }} \\
(+90 \mathrm{~K})\end{array}$ & $\begin{array}{c}\Delta \log g \\
(+0.20 \mathrm{dex})\end{array}$ & $\begin{array}{c}\Delta[\mathrm{M} / \mathrm{H}] \\
(+0.10 \mathrm{dex})\end{array}$ & $\begin{array}{c}\Delta \xi \\
\left(+0.25 \mathrm{~km} \mathrm{~s}^{-1}\right)\end{array}$ & $\sigma$ \\
\hline $3850 \mathrm{~K} ; 1.20 ; 0.00$ & +0.04 & +0.08 & +0.02 & -0.05 & $\overline{0.10}$ \\
\hline $3990 \mathrm{~K} ; 0.53 ;-1.15$ & +0.05 & +0.08 & +0.04 & -0.02 & 0.10 \\
\hline
\end{tabular}

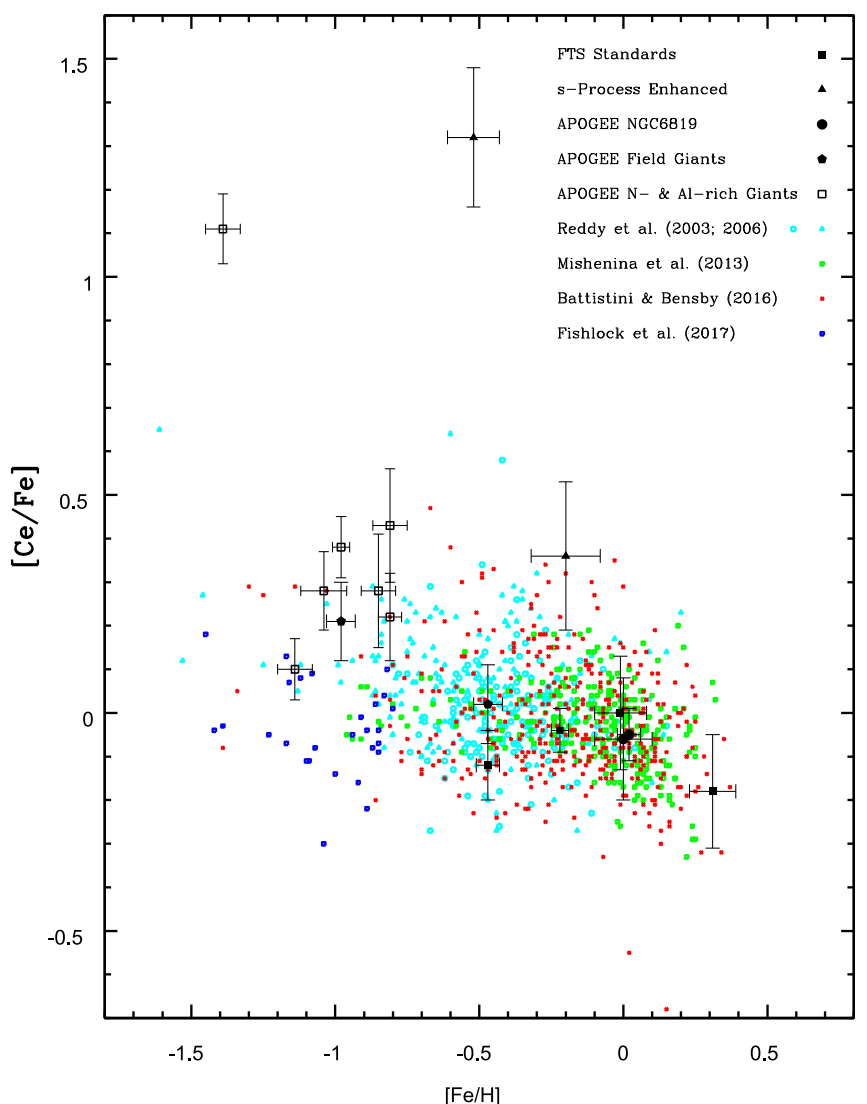

Figure 7. $[\mathrm{Ce} / \mathrm{Fe}]$ vs. $[\mathrm{Fe} / \mathrm{H}]$ for the stars analyzed here (black symbols), along with samples of field stars from Reddy et al. (2003, 2006), Mishenina et al. (2013), Battistini \& Bensby (2016), and Fishlock et al. (2017). The error bars correspond to the standard deviations of the mean abundances. The black triangles indicate stars that were previously known to be enhanced in s-process elements. The solar values for $\mathrm{Ce}$ and $\mathrm{Fe}$ are from Grevesse et al. (2015) and Asplund et al. (2005), respectively.

that compare well with those abundances derived from optical $\mathrm{Ce}$ II lines in the high metallicity regime $([\mathrm{Fe} / \mathrm{H}]>-0.7)$. At lower metallicities, a larger sample of stars with "normal" chemistry is needed in order to verify possible systematic differences between the $\mathrm{Ce}$ abundance results from the optical and the infrared.

\subsection{Abundance Sensitivity of the $\lambda 16376 \AA$ Line Across Stellar Parameter Space}

The synthetic and observed APOGEE spectra shown in Figures 5 and 6 demonstrate that the Ce II lines are easily detectable and can be modeled well at the resolution of APOGEE not only in red giants that have stellar parameters similar to red clump stars at solar metallicity (the NGC 6819 giants), but also in cooler, moderately metal-poor red giants (such as 2M15203732-0207263; [Fe/H] -1.0). To roughly estimate the parameter space where the Ce II would be detectable and measurable in the spectra of typical red giants

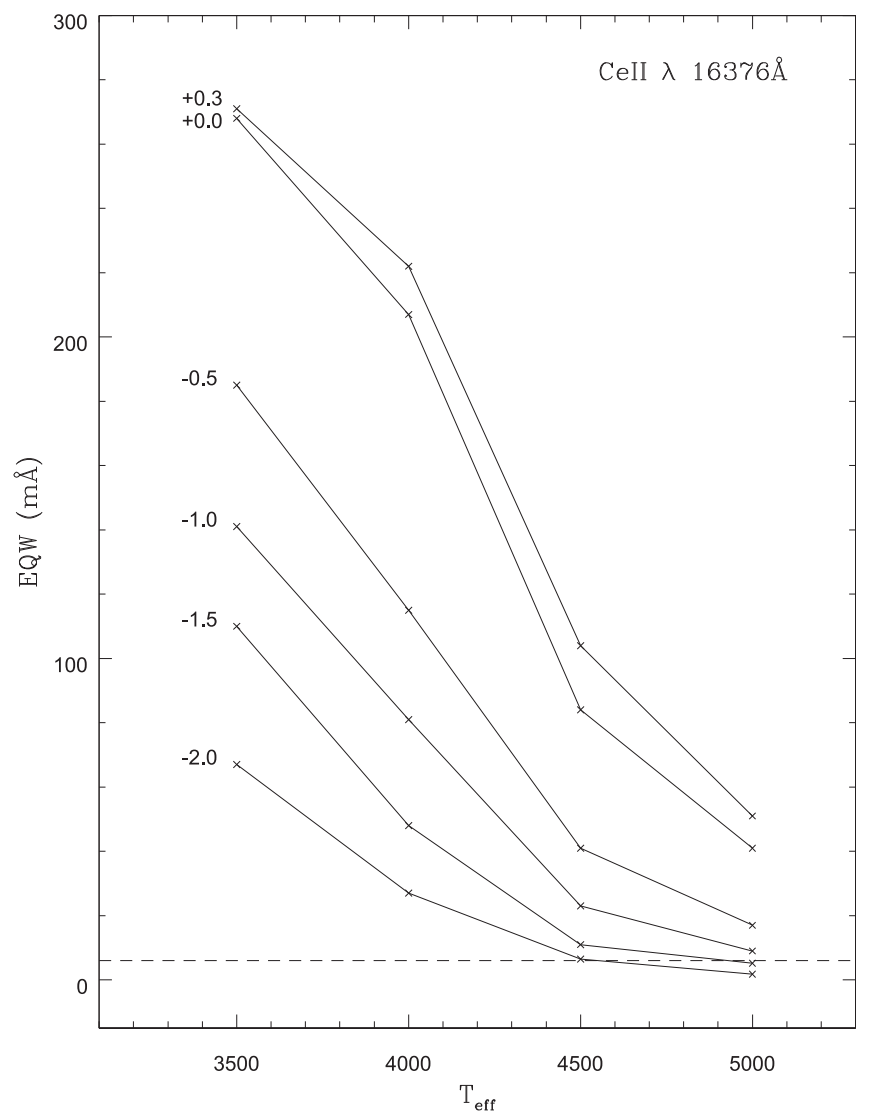

Figure 8. Equivalent width of the $\lambda 16376 \AA$ Ce II line as a function of $T_{\text {eff }}$ and $[\mathrm{Fe} / \mathrm{H}]$, with each value of $T_{\text {eff }}$ paired with a $\log g$ value typical for a red giant $\left(T_{\text {eff }}=3500 \mathrm{~K}, \quad \log g=0.5 ; \quad T_{\text {eff }}=4000 \mathrm{~K}, \quad \log g=1.0 ; \quad T_{\text {eff }}=4500 \mathrm{~K}\right.$, $\log g=1.5$; and $T_{\text {eff }}=5000 \mathrm{~K}, \log g=2.0$ ). The Ce II equivalent widths are calculated assuming $[\mathrm{Ce} / \mathrm{Fe}]=0.0$. This $\mathrm{Ce}$ II line will be detectable in a significant fraction of APOGEE red giants. It falls below detection limits only in the hotter and quite metal-poor red giants. The horizontal dashed line indicates the approximate equivalent width detection of $\sim 6 \mathrm{~m} \AA$ for an APOGEE spectrum with an $\mathrm{S} / \mathrm{N}=100$.

observed by the APOGEE survey, one of the stronger Ce II lines was used as a test case to map its equivalent width over a range of stellar parameters. A simple, visual representation of the strength of this Ce II line is presented in Figure 8, where the equivalent width of the $\lambda 16376 \AA$ line is plotted versus $T_{\text {eff }}$ for a range of metallicities (from $[\mathrm{Fe} / \mathrm{H}]=+0.3$ down to -2.0 ). Each $T_{\text {eff }}$ value is paired with a $\log g$ that is typical for a lowmass red giant of that effective temperature (log $g$ decreasing from 2.5 at $5000 \mathrm{~K}$ to 0.5 at $3500 \mathrm{~K}$ ).

Given the resolution of APOGEE $(R \sim 22,500)$ and its pixel sampling $(\Delta \lambda=0.22 \AA)$, the detection limit in the equivalent width of a line is about $6 \mathrm{~mA}$ (denoted by the dashed horizontal line in Figure 8) for an $\mathrm{S} / \mathrm{N}$ of 100. Although typical spectral lines are all blended to various degrees in red giant APOGEE spectra, Figure 8 remains a valid visual aid in estimating where the Ce II lines will be most detectable in the observed red giant 


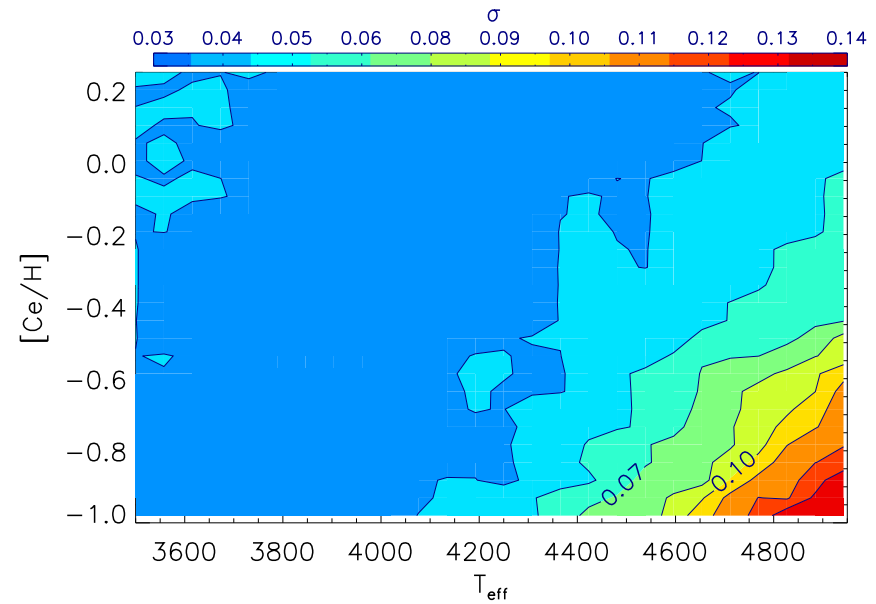

Figure 9. Contour plot of the uncertainties (in dex) of $[\mathrm{Ce} / \mathrm{H}]$ as a function of $T_{\text {eff }}$ and $\log g$ (with each $T_{\text {eff }}$ paired with a corresponding $\log g$ along the red giant branch; same as Figure 8). The calculations are done for the $\lambda 16376 \AA$ Ce II line, the same Ce II line as used in Figure 8, and the APOGEE spectra with $\mathrm{S} / \mathrm{N}=100$.

populations. Not surprisingly, the hottest and most metal-poor red giants will be the most difficult stars in which to probe cerium abundances, while the cooler red giants will yield abundances from Ce II down to quite low metallicities. Of course, for s-process enhanced stars, with $[\mathrm{Ce} / \mathrm{Fe}]>0$, detecting cerium will be even easier.

Related to the ability to detect and measure the Ce II lines in red giant spectra, as illustrated in Figure 8, is the uncertainty in synthesizing a blended Ce II line (at the APOGEE resolution) as shown in Figure 9. Here, uncertainties in determining values of $[\mathrm{Ce} / \mathrm{H}](\delta \mathrm{Ce})$ are plotted as contours in a $[\mathrm{Ce} / \mathrm{H}]$ versus $T_{\text {eff }}$ plane. Each value of $T_{\text {eff }}$ is paired with a corresponding $\log g$ following a typical RGB track at each respective metallicity (these calculations were done assuming $[\mathrm{Ce} / \mathrm{Fe}]=0.0$ ). Each uncertainty point is calculated as the $\delta \mathrm{Ce}$ abundance (in dex) required to change the relative flux by 0.01 some $0.2 \AA$ from the line center for the Ce II $\lambda 16376 \AA$ line in synthetic APOGEE spectra. The change in flux level by 0.01 is the noise level for an APOGEE spectrum with $\mathrm{S} / \mathrm{N}=100$. This indicates that the parameter space over which this Ce II line can be measured in the APOGEE spectra covers all stars cooler than $\sim 4700 \mathrm{~K}$ down to at least $[\mathrm{Fe} / \mathrm{H}]=-1.0$. Above $4700 \mathrm{~K}$, this Ce II line will be detectable in stars more metal rich than $\sim-0.6$ dex.

\section{Conclusions}

The focus of this study was to investigate the spectral APOGEE window (between $\lambda 1.51-1.69 \mu \mathrm{m}$ ) for potential atomic lines arising from elements produced by the s-process. A number of previously unidentified lines were found to be singly ionized transitions of the s-process element cerium. Nine Ce II lines were identified and characterized in the spectra of a sample of red giants observed with the FTS on the KPNO $4 \mathrm{~m}$ telescope and APOGEE. In the absence of laboratory measurements for the $g f$-values of these transitions, astrophysical $g f$-values were derived using the well-studied K-giant $\alpha$ Boo as a standard star, along with the solar spectrum used for identifying and defining other blending lines. The astrophysical $g f$-values depend on the cerium abundance for $\alpha$ Boo that was derived here from a sample of optical Ce II lines with accurate $g f$-values from Lawler et al. (2009); the $g f$-values presented here are thus tied to this laboratory scale. Future laboratory measurements of $g f$-values for the Ce II lines in the APOGEE window would be a valuable addition.

The usefulness of this set of Ce II lines in future abundance studies in the APOGEE wavelength region was evaluated using a sample of red giants covering a range of effective temperatures, surface gravities, and metallicities. Taken together, the combination of high-resolution FTS spectra of a few well-studied red giant stars, including the APOGEE standard $\alpha$ Boo, coupled with a metal-poor, very s-process enriched red giant, along with a small number of APOGEE red giants with normal s-process abundances, allowed for a thorough mapping of the Ce II lines within the APOGEE spectral region. The analyses of seemingly normal red giants observed by APOGEE indicated that the Ce II lines can be reliably used for cerium abundances across a significant portion of the APOGEE red giants and, in particular, in those stars that are cooler than $\sim 4700 \mathrm{~K}$ down to $[\mathrm{Fe} / \mathrm{H}] \sim$ -1.0 . For hotter stars, cerium abundances can be measured for metallicities above $[\mathrm{Fe} / \mathrm{H}] \sim-0.6$. For the cooler red giants $\left(T_{\text {eff }}\right.$ less than $\left.\sim 4000 \mathrm{~K}\right)$, Ce II should be detectable well below $[\mathrm{Fe} / \mathrm{H}]=-2.0$.

This is the first study to discover and characterize these particular Ce II lines that fall in the APOGEE spectral region. The results from this paper will be added to the APOGEE line list for a future SDSS data release and will impact the APOGEE survey as it opens the study of a representative s-process element in regions of the Milky Way that are only accessible in the infrared. A companion paper presenting results for Nd II lines is that of Hasselquist et al. (2016).

We thank the referee for suggestions that improved the paper. V.V.S. acknowledges partial support for this research from the National Science Foundation (AST1109888). D.A.G.H. was funded by the Ramón y Cajal fellowship number RYC201314182, and D.A.G.H. and O.Z. acknowledge support provided by the Spanish Ministry of Economy and Competitiveness (MINECO) under grant AYA201458082-P. Sz.M. has been supported by the Premium Postdoctoral Research Program of the Hungarian Academy of Sciences, and by the Hungarian NKFI Grants K-119517 of the Hungarian National Research, Development and Innovation Office. This publication makes use of data products from the Two Micron All Sky Survey, which is a joint project of the University of Massachusetts and the Infrared Processing and Analysis Center/California Institute of Technology, funded by the National Aeronautics and Space Administration and the National Science Foundation. This research has made use of the SIMBAD database, operated at CDS, Strasbourg, France. C.A.P. is thankful to the Spanish MINECO for support under grant AYA2014-56359-P. H.J. acknowledges support from the Birgit and Hellmuth Hertz' Foundation, the Royal Physiographic Society of Lund, Sweden.

Funding for the Sloan Digital Sky Survey IV has been provided by the Alfred P. Sloan Foundation, the U.S. Department of Energy Office of Science, and the Participating Institutions. SDSS-IV acknowledges support and resources from the Center for High-Performance Computing at the University of Utah. The SDSS Web site is www.sdss.org.

SDSS-IV is managed by the Astrophysical Research Consortium for the Participating Institutions of the SDSS 
Collaboration including the Brazilian Participation Group, the Carnegie Institution for Science, Carnegie Mellon University, the Chilean Participation Group, the French Participation Group, Harvard-Smithsonian Center for Astrophysics, Instituto de Astrofísica de Canarias, The Johns Hopkins University, Kavli Institute for the Physics and Mathematics of the Universe (IPMU)/University of Tokyo, Lawrence Berkeley National Laboratory, Leibniz Institut für Astrophysik Potsdam (AIP), Max-Planck-Institut für Astronomie (MPIA Heidelberg), Max-Planck-Institut für Astrophysik (MPA Garching), MaxPlanck-Institut für Extraterrestrische Physik (MPE), National Astronomical Observatory of China, New Mexico State University, New York University, University of Notre Dame, Observatório Nacional/MCTI, The Ohio State University, Pennsylvania State University, Shanghai Astronomical Observatory, United Kingdom Participation Group, Universidad Nacional Autónoma de México, University of Arizona, University of Colorado Boulder, University of Oxford, University of Portsmouth, University of Utah, University of Virginia, University of Washington, University of Wisconsin, Vanderbilt University, and Yale University.

Facilities: SDSS, Kitt Peak.

\section{References}

Allende Prieto, C., Beers, T. C., Wilhelm, R., et al. 2006, ApJ, 363, 804 Asplund, M., Grevesse, N., \& Sauval, A. J. 2005, in ASP Conf. Ser. 336, Cosmic Abundances as Records of Stellar Evolution and Nucleosynthesis, ed. T. G. Barnes III \& F. N. Bash (San Francisco, CA: ASP), 25

Battistini, C., \& Bensby, T. 2016, A\&A, 586, A39

Blaise, J., Chevillard, J., Verges, J., \& Wyart, J. F. 1970, AcSpe, 25, 333

Blanton, M., Bershady, M., Anderson, S., et al. 2017, AJ, 154, 28

Bressan, A., Marigo, P., Girardi, L., et al. 2012, MNRAS, 427, 127

Corliss, C. H. 1973, JRNBS, 77A, 419

Cunha, K., Smith, V. V., Johnson, J. A., et al. 2015, ApJL, 798, L41

Davis, S. P., \& Phillips, J. G. 1963, The Red System of CN Molecule (Berkeley, CA: Univ. California Press)

Eisenstein, D. J., Weinberg, D. H., Agol, E., et al. 2011, AJ, 142, 72

Fernández-Trincado, J. G., Robin, A. C., Moreno, E., et al. 2016, ApJ, 833,132

Fishlock, C. K., Yong, D., Karakas, A., et al. 2017, MNRAS, 466, 4672
Fulbright, J. P., McWilliam, A., \& Rich, R. M. 2007, ApJ, 661, 1152 García Pérez, A. E., Allende Prieto, C., Holtzman, J. A., et al. 2016, AJ, 151,144

Grevesse, N., Scott, P., Asplund, M., \& Sauval, A. J. 2015, A\&A, 573, 27

Gunn, J. E., Siegmund, W. A., Mannery, E. J., et al. 2006, AJ, 131, 2332

Hasselquist, S., Shetrone, M., Cunha, K., et al. 2016, ApJ, 833, 81

Hinkle, K., Wallace, L., \& Livingston, W. 1995, PASP, 107, 1042

Hinkle, K., Wallace, L., Valenti, J., \& Harmer, D. 2000, Visible and Near Infrared Atlas of the Arcturus Spectrum 3727-9300 A (San Francisco, CA: ASP)

Holtzman, J. A., Shetrone, M., Johnson, J. A., et al. 2015, AJ, 150, 148

Kurucz, R. L. 1993, ATLAS9 Stellar Atmosphere Programs and $2 \mathrm{~km}$ sE49ACD1 grid. Kurucz CD-ROM No. 13 (Cambridge, MA: Smithsonian Astrophysical Observatory)

Lawler, J. E., Sneden, C., Cowan, J. J., Ivans, I. I., \& Den Hartog, E. A. 2009, ApJS, 182, 51

Livingston, W., \& Wallace, L. 1991, An Atlas of the Solar Spectrum in the Infrared from 1850 to $9000 \mathrm{~cm}^{-1}$ (1.1 to 5.4 micrometer), NSO Technical Rep. (Tucson, AZ: National Solar Observatory)

Lodders, K. 2010, ASSP, 16, 379

Majewski, S., Schiavon, R. P., Frinchaboy, Peter M., et al. 2017, AJ, submitted Mészáros, S., Allende Prieto, C., Edvardsson, B., et al. 2012, AJ, 144, 120

Mishenina, T. V., Pignatari, M., Korotin, S. A., et al. 2013, A\&A, 552, A128

Nidever, D. L., Holtzman, J. A., Allende Prieto, C., et al. 2015, AJ, 150, 173

Pereira, C. B., Smith, V. V., Drake, N. A., et al. 2017, MNRAS, 469, 774

Ramirez, I., \& Allende Prieto, C. A. 2011, ApJ, 743, 135

Reddy, B. E., Lambert, D. L., \& Allende Prieto, C. 2006, MNRAS, 367, 1329

Reddy, B. E., Tomkin, J., Lambert, D. L., \& Allende Prieto, C. 2003, MNRAS, 340, 304

Ryde, N., Gustafsson, B., Edvardsson, B., et al. 2010, A\&A, 509, A20

Schiavon, R. P., Johnson, J. A., Frinchaboy, P. M., et al. 2017a, MNRAS, 466, 1010

Schiavon, R. P., Zamora, O., Carrera, R., et al. 2017b, MNRAS, 465, 501

Shetrone, M., Bizyaev, D., Lawler, J. E., et al. 2015, ApJS, 221, 24

Smith, V. V., Cunha, K., Shetrone, M. D., et al. 2013, ApJ, 765, 16

Smith, V. V., \& Lambert, D. L. 1990, ApJS, 72, 387

Sneden, C. 1973, ApJ, 184, 839

Sneden, C., Cowan, J. J., \& Gallino, R. 2008, ARA\&A, 46, 241

Wahlgren, G. M., Nave, G., Nilsson, H., \& Lundqvist, M. 2008, JPhCS, 130, 012019

Wilson, J. C., Hearty, F., Skrutskie, M. F., et al. 2010, Proc. SPIE, 7735, $77351 \mathrm{C}$

Zamora, O., Garcia-Hernandez, D. A., Allende Prieto, C., et al. 2015, AJ, 149,181

Zasowski, G., Johnson, J. A., Frinchaboy, P. M., et al. 2013, AJ, 146, 81 